\title{
Einige Überlegungen zur strategischen Ausrichtung des Lehrstuhls für Aufbereitung und Veredlung in Zeiten einer sich rasant verändernden Bildungs- und Forschungslandschaft
}

\author{
Helmut Flachberger \\ Lehrstuhl für Aufbereitung und Veredlung, Department Mineral Resources Engineering, Montanuniversität \\ Leoben, Leoben, Österreich
}

Eingegangen 30. Oktober 2020; angenommen 31. Oktober 2020; online publiziert 16. November 2020

\begin{abstract}
Zusammenfassung: Die Berufung von Prof. Bierbrauer zum ersten Ordinarius der kurz zuvor eingerichteten Lehrkanzel für Aufbereitung an der Montanistischen Hochschule Leoben erfolgte mit Oktober 1930, also genau vor 90 Jahren. Diesen runden Geburtstag möchte der Verfasser dieser ZeiIen, der den Lehrstuhl von seinem akademischen Lehrer Prof. Hans Jörg Steiner - im September 2005 übernahm, zum Anlass nehmen, auf Basis eines Blickes in die Vergangenheit und einer Umfeldanalyse mögliche Wege für eine gedeihliche Weiterentwicklung dieses Fachbereiches zu skizzieren.
\end{abstract}

Der Blick zurück in die jüngste Vergangenheit zeigt eindrucksvoll auf, wie sehr sich die österreichische Universitätslandschaft durch das Universitätsgesetz 2002 (UG) verändert hat und welche Konsequenzen sich daraus ergeben haben. Die Montanuniversität Leoben wurde in die Autonomie entlassen, neue Leitungsstrukturen und Organisationseinheiten wurden eingeführt, um nur einige Eckpfeiler dieses Gesetzes anzuführen. Nahezu zeitgleich wurde mit der Umsetzung des Bologna-Prozesses mit dem übergeordneten Ziel der Verbesserung der Mobilität von Lehrenden und Lernenden begonnen und damit die altbewährten Diplomstudien in Bachelor- und Masterstudien untergliedert. Die Fachhochschulen, oftmals gut dotiert und damit auch gut ausgestattet, haben eine große Vielzahl an Studiengängen aufgebaut, die es mit Studierenden auszulasten gilt, die sie sich noch dazu "aussuchen“ dürfen. Die Rohstoffbranche wurde vom rauen Wind der Wirtschaftskrise z. T. schwer getroffen, was über einige Jahre hinweg sinkende Forschungsausgaben und Einschränkungen bei

\footnotetext{
Univ. Prof. Dipl.-Ing. Dr. mont. H. Flachberger ( $₫)$

Lehrstuhl für Aufbereitung und Veredlung, Department Mineral

Resources Engineering,

Montanuniversität Leoben,

Franz-Josef-Str. 18,

8700 Leoben, Österreich

helmut.flachberger@unileoben.ac.at
}

der Mitarbeiterentwicklung mit sich brachte. Die seitens des Rektorates verfolgte Campusstrategie hat die Zusammenführung von fachnahen Lehrstühlen „unter ein Dach“ zur Folge, was das Verlassen des Rittinger-Gebäudes und das Besiedeln des Impulszentrums für Rohstoffe (IZR) mit sich brachte.

Am 01.01.2006 wurde aus dem Institut für Aufbereitung und Veredlung der Lehrstuhl für Aufbereitung und Veredlung und dieser dem Department Mineral Resources and Petroleum Engineering, mit 01.01.2015 dem Department Mineral Resources Engineering zugeordnet. Die Besiedelung des IZR erfolgte im 2. Quartal 2011.

Der Lehrstuhl für Aufbereitung und Veredlung reagierte und reagiert auf diese vielfältigen An- und Herausforderungen durch eine fortlaufend überdachte Ausrichtung und Anpassung in Lehre und Forschung. Beispielhaft sei die mit 01.10.2015 in Kraft getretene Umstellung (von einem bislang dreisemestrigen) auf ein nunmehr viersemestriges Schwerpunktfach Aufbereitung und Veredlung im Masterstudium Rohstoffverarbeitung, welche eine vertiefende Ausbildung künftiger Aufbereiter*innen ermöglicht.

In der Lehre werden vorrangig das Bachelorstudium Rohstoffingenieurwesen, das Schwerpunktfach Aufbereitung und Veredlung im Masterstudium Rohstoffverarbeitung wie auch das Bachelor- und Masterstudium Recyclingtechnik, das mit dem Wintersemester 2014/15 eingerichtet wurde, betreut. Darüber hinaus werden aufbereitungstechnische Lehrveranstaltungen für eine ganze Reihe weiterer Studien abgehalten. Mit dem Studienjahr 2013/14 wurde ein neuer, berufsbegleitender Universitätslehrgang Rohstoffaufbereitung eingerichtet. Der mittlerweile vierte Jahrgang wurde kürzlich gestartet.

Es wurden drei Hauptforschungsfelder definiert - die konventionelle Mineralaufbereitung, die Sekundärrohstoffaufbereitung und die Funktionsmineralaufbereitung - die den fachlichen Rahmen für eine anwendungsorientierte und experimentell ausgerichtete Forschung auf hohem Niveau bil- 
den sollen. Aus den überwiegend gemeinsam mit Industriepartnern abgewickelten Forschungsaktivitäten werden einige Entwicklungstendenzen ohne Anspruch auf Vollständigkeit abgeleitet.

Schlüsselwörter: Aufbereitung und Veredlung, Montanuniversität Leoben, Lehr- und Forschungsaktivitäten

Some Reflections on the Strategic Orientation of the Chair of Mineral Processing in Times of a Rapidly Changing Education and Research Environment

Abstract: The appointment of Prof. Bierbrauer as first full professor of the previously established Institute for Mineral Processing at the Montanistische Hochschule Leoben took place in October 1930, exactly 90 years ago. The author of these lines, who took over the chair from his academic teacher - Prof. Hans Jörg Steiner - in September 2005, would like to take this milestone birthday occasion to outline possible ways for a prosperous further development of this chair on the basis of a look into the past and an analysis of the present development.

A look back into the recent past impressively shows how much the Austrian university landscape has changed as a result of the University Act 2002 and what consequences have resulted from this. The Montanuniversitaet Leoben was released into autonomy, new management structures and organizational units were introduced, to name just a few cornerstones of this law. Almost simultaneously, the implementation of the Bologna Process was started with the overriding goal of improving the mobility of teachers and students and thus the well-tried diploma studies were substituted by Bachelor and Master studies. The universities of applied sciences, often well funded and thus well equipped, have built up a large number of study programs that need to be filled with students they can select by themselves. The raw materials industry was in some cases severely affected by the harsh winds of the economic crisis, which resulted in declining research spendings and restrictions on staff development for several years. The campus strategy pursued by the Rectorate resulted in the merging of subject-related chairs "under one roof", which meant leaving the Rittinger building and settling in the Impulse Center for Raw Materials (IZR).

On January 1, 2006, the Chair of Mineral Processing was merged with six other chairs into the Department of Mineral Resources and Petroleum Engineering and on January 1, 2015 in the Department of Mineral Resources Engineering. The settlement of the IZR took place in the 2 nd quarter of 2011.

The Chair of Mineral Processing has been responding and continues to respond to these diverse demands and challenges by continuously rethinking and adapting its teaching and research activities. One example is the changeover from a three-semester course to a four-semester specialization in the main subject Mineral Processing in the Master's program in Raw Materials Engineering, which came into effect on October 1, 2015, and which will enable a more indepth education of future mineral processors.
Teaching is primarily focused on the bachelor's degree program in Mineral Resources Engineering, the specialization in Mineral Processing in the master's degree program in Raw Materials Processing, and the bachelor's and master's degree programs in Recycling Technology, which was established in the winter semester 2014/15. In addition, courses in process technology are offered in a wide range for other studies. With the 2013/14 academic year, a new part-time university course in Raw Material Processing was established. The fourth class has recently been launched. Three main fields of research were defined-conventional mineral processing, secondary raw material processing and functional mineral processing-which are to form the technical framework for application-oriented and experimentally oriented research at a high level. From the research activities, which are mainly carried out together with industrial partners, some development trends are derived without claiming to be complete.

Keywords: Mineral processing, Montanuniversitaet Leoben, Education and research activities

\section{Einleitung}

Um es gleich zu Beginn in klaren Worten niederzuschreiben: In den kommenden Jahren wird in der österreichischen Bildungs- und Forschungslandschaft kein Stein auf dem anderen bleiben.

Die Gründe dafür sind vielfältiger Natur. Ohne Anspruch auf Vollständigkeit seien

- aufkeimende Initiativen zur Bildung eines europäischen Hochschulraumes (Stichwort: European University),

- die Gründung neuer Universitäten (Stichwort: TU Linz) und das örtliche, fachliche oder formale Zusammenführen bestehender Universitäten (Stichwort: Kooperationsprojekt Naturwissenschaften (NAWI) Graz, TU Austria),

- die enorme Diversifizierung der österreichischen (Aus-)Bildungslandschaft (Stichwort: Fachhochschulen mit ihren vielen Studiengängen),

- die leistungsbezogene Dotierung der Bildungseinrichtungen (Stichwort: prüfungsaktive Studierende),

- der politische Druck zu einer rasanten Akademisierung der Bevölkerung (Stichwort: OECD-Report),

- der Druck der Industrie nach einer Verkürzung von Ausbildungszeiten (Stichwort: IV-Positionspapier),

- das Bestreben des Ministeriums für Bildung, Forschung und Wissenschaft nach einer Erhöhung der Durchlässigkeit zwischen den (Aus-)Bildungseinrichtungen (Stichwort: vom Meister zum Master),

- und der immer härter ausgetragene „Kampf“ um die besten Köpfe aus den jeweiligen Maturant*innen-Jahrgängen (Stichwort: geburtenschwache Jahrgänge).

angeführt. 


\section{Umfeldbetrachtung}

\subsection{Europäische Impulse}

Die vergangenen zwei Jahrzehnte waren geprägt von der Umsetzung des Bologna-Prozesses und der damit einhergehenden Umgestaltung der bislang etablierten Diplomstudien in Bachelor- und Masterstudien. Die Montanuniversität Leoben (nachfolgend mit MUL abgekürzt) hat diesen Prozess früh begonnen und in mehreren Etappen abgeschlossen. Die zwölf an der MUL angebotenen Bachelorstudien sind mittlerweile einheitlich sieben-semestrig, wobei in Summe 240 ECTS-Punkte (European Credit Transfer and Accumulation System) vergeben werden und ein Semester der Absolvierung einer verpflichtenden Praxis zugeordnet wurde. Die Absolvent*innen schließen mit dem akad. Grad "Bachelor of Science“ (abgekürzt BSc) ab. Die dreizehn an der MUL angebotenen Masterstudien sind mittlerweile einheitlich vier-semestrig, wobei in Summe 120 ECTS-Punkte vergeben werden und das vierte Semester dem Schreiben der Masterarbeit gewidmet ist. Die Absolvent*innen schlieBen nach wie vor mit dem akad. Grad "Diplom-Ingenieur" (abgekürzt Dipl.-Ing. oder DI) ab.

Eine ebenso wichtige europäische Initiative mit Auswirkungen auf Österreich und die MUL war das Umdenken der Europäischen Kommission rund um die Themen "Rohstoffe" bzw. "Rohstoffbeschaffung". So verabschiedete die EU im Dezember 2015 ein ehrgeiziges Maßnahmenpaket zur Kreislaufwirtschaft, um die Wettbewerbsfähigkeit zu steigern, Arbeitsplätze zu schaffen und ein nachhaltiges Wachstum zu erreichen. Ein Teil der geforderten Aktionspläne wurde in den letzten Jahren bereits umgesetzt, allerdings werden die geforderten Ziele erst durch enorme Anstrengungen im nächsten Jahrzehnt erreicht werden können. Jedenfalls führte die Steigerung der Bedeutung der Eigenversorgung mit (kritischen) mineralischen Rohstoffen und des Wiederverwertens von Reststoffen unserer Wohlstandsgesellschaft durch Recycling bereits jetzt zu mannigfaltigen forschungsrelevanten Akzenten bei diversen Förderprogrammen - EU-weit z. B. in der Programmschiene "Horizon 2020 (H2020)“, in Österreich z. B. durch die Programmschiene "Produktion der Zukunft" - wie auch zur Gründung des EIT RawMaterials (European Institute of Innovation and Technology RawMaterials), dem die MUL seit Anbeginn angehört, maßgeblich an dessen Aufbau beteiligt war und durch das sich viele positive Entwicklungen ergaben. Auch die Gründung des Helmholtz-Institutes Freiberg für Ressourcentechnologie an der TU Bergakademie Freiberg ist das Ergebnis einer dieser Initiativen auf deutschem Boden.

Nicht unerwähnt bleiben darf der Zusammenschluss von zuvor eher national geprägten Rohstoff-Fachverbänden (z. B. die Fachverbände "Stein- und keramische Industrie“ und „Bergwerke und Stahl“) zu europäischen Rohstoffverbänden, wie z. B. den Europäischen Gesteinsverband UEPG und EUROMINES bzw. EUMICON, die wesentliche Impulse zu einem verbesserten Verständnis der strategischen Bedeutung der eigenständigen Rohstoffversorgung gesetzt haben.

\subsection{Nationale Impulse}

Das Universitätsgesetz 2002 mit all den nachfolgenden Novellen hat die österreichische Universitätslandschaft grundlegend verändert. Das Entlassen der Universitäten in die Autonomie wurde als befreiend empfunden und hat viele Initiativen gefördert. Auch die Montanuniversität hat diese Chance genutzt, um ihr Bildungs- und Forschungsprofil entlang des Wertschöpfungskreislaufes zu verstärken bzw. zu komplettieren, beispielsweise durch den Aufbau der Studienrichtungen "Industrielogistik“, "Recyclingtechnik“ und jüngst „Industrial Data Science.. Als nachteilige Entwicklung ist zu nennen, dass der durch die Autonomie sich ergebende "nachlassende ministerielle Einfluss“ zur Erstellung einiger "Raubkopien“ von montanistischen Kernfächern geführt hat, als Beispiele seien die Einrichtung werkstoffwissenschaftlicher Studien an der Universität Salzburg oder kunststofftechnischer Studien an der Johannes Kepler Universität (JKU) Linz angeführt.

Die drei technischen Universitäten Österreichs - die TU Wien, die TU Graz und die Montanuniversität Leoben - haben sich zur TU Austria zusammengeschlossen. Sie folgten dabei dem Trend mehrerer Länder der EU, deren Universitäten zu Universitäts-Verbunden zusammenzuschließen, wie etwa die TU7 in Deutschland. Die Zielsetzungen sind mannigfaltiger Natur und reichen von einer Abstimmung bei fachlichen Schwerpunkten bis zu gemeinsamen Anschaffungen bei teuren Forschungsaggregaten. An der TU Austria wurde zum Beispiel beschlossen, in den Bereichen „Tunnelbau“ und „Energietechnik" gemeinsame Initiativen zu fördern.

Eine Exzellenzinitiative für Österreichs Universitäten ist zwar seit mehreren Jahren angekündigt, eine Umsetzung steht derzeit aber noch aus. In manchen Ländern führten Exzellenzinitiativen zu einem hoch kompetitiven universitären Umfeld, was zu einer Schärfung des Profils und zur Bildung universitätsübergreifender Forschungs-Cluster führte.

Der österreichische "Rohstoffplan“ ist die rundum gelungene "österreichische Antwort" auf die steigende Bedeutung der Sicherung der Eigenversorgung für mineralische Rohstoffe mit einer gewissen Schwerpunktsetzung auf Baurohstoffe. Die Implementierung ist erfolgt und wurde überwiegend positiv angenommen.

Derzeit arbeitet das Bundesministerium an der Rohstoffstrategie 2030, in deren Erarbeitung die MUL stark eingebunden ist. In diesem Zusammenhang erfolgt eine gesamtheitliche Betrachtung der primären und sekundären $\mathrm{Ge}$ stehungsprozesse, wobei auch jene Abfälle (Nebenprodukte), welche bei den Erzeugungsprozessen entstehen, besondere Berücksichtigung finden. Darüber hinaus werden Querschnittsthemen wie Bildung, Digitalisierung, rechtliche Rahmenbedingungen $u$.a. behandelt, um eine nachhaltige Rohstoffversorgung in Österreich zu garantieren.

\section{Die Montanuniversität Leoben}

Die Montanuniversität Leoben (MUL) hat es bislang verstanden, sich den Herausforderungen der jüngsten Zeit mutig zu stellen und zukunftsträchtige Konzepte zu ent- 
wickeln, um ihren Fortbestand zu sichern. Die geglückte Umsetzung des Bologna-Prozesses und die konsequente Erweiterung der Bildungs- und Forschungsfelder entlang des Wertschöpfungskreislaufes - vom primären Rohstoff, zum Werkstoff, zum Reststoff, zum sekundären Rohstoff und wiederum zum Produkt - und die damit einhergehende Profilbildung seien als positive Beispiele angeführt.

Gemeinsam mit den Kompetenzzentren - Materials Center Leoben (MCL), Polymer Competence Center Leoben (PCCL), K1Met, Erich-Schmidt-Institut der Österreichischen Akademie der Wissenschaften, Österreichisches GießereiInstitut - arbeiten etwa 2000 Forscher*innen in und um Leoben in montanistischen Kernfächern, was zu einer guten internationalen Sichtbarkeit in der Scientific Community beiträgt. Beispielhaft ist in diesem Zusammenhang die TopPlatzierung des Fachbereiches "Mining and Minerals Engineering" (weltweit Nummer 37, in Europa Nummer 5) beim fachbezogenen Shanghai-Ranking zu sehen, wodurch die hochqualitative Forschung im Besonderen herausgestrichen wird. Das vergleichsweise gute Betreuungsverhältnis führt bei Studierendenbefragungen regelmäßig zu TopPlatzierungen bei diversen Rankings und die lebenslange Verbundenheit unserer Absolvent*innen sucht ihresgleichen. Die enge Kooperation mit der nahestehenden Industrie führt zur Einwerbung von Drittmittelvolumina, die an jene, seitens des Bundes zur Verfügung gestellten Globalbudgets herankommen.

Diesen positiven Entwicklungen stehen die vergleichsweise geringe Attraktivität wie auch Bekanntheit unserer Alma Mater Leobiensis unter den Studierwilligen gegenüber. Unsere Studien werden als (zu) schwierig, die Studienzeiten als (zu) lange empfunden und über diverse Social-Media-Kanäle „spricht sich das rasch herum" (Anm.: egal, ob diese Empfindung stimmt, oder nicht). Die Botschaft, dass es bestens ausgebildeter Ingenieur*innen bedarf, um die Herausforderungen unserer Weltgemeinschaft in den Themen „Umwelt”, "Energie“, „Klima“ zu meistern, und dass "wir in Leoben“ uns mit genau diesen Themenstellungen auseinandersetzen, wird nicht gehört. Mit den glänzenden Berufsaussichten ergeht es uns gleich. Diese Schwierigkeiten führen dazu, dass wir seit 2015 - wo an der MUL erstmals mehr als 4000 Studierende ausgebildet wurden - mit sinkenden Hörer*innenzahlen zu kämpfen haben, was uns u. a. aus Sicht des Globalbudgets in Anbetracht der künftig vornehmlich leistungsbezogenen Dotierung recht rasch in Schwierigkeiten bringen kann.

\subsection{Das Department Mineral Resources Engineering}

Mit der Departmentgründung nahm der Rohstoffbereich innerhalb der MUL eine Vorreiterrolle ein. Das gemeinsame Vertreten der Interessen der Lehrstühle und das geschlossene Auftreten als Department haben sich als ausgesprochen wichtig und vorteilhaft erwiesen. So wurden die verstreut situierten Lehrstühle im Zuge der Campusstrategie des damaligen Rektorates nicht nur organisatorisch, sondern auch örtlich "(fast) unter ein Dach“ zusammengeführt. Drei der vier Lehrstühle sind seitdem mit ihren Office-
Bereichen in den unterschiedlichen Ebenen des Rohstoffund Werkstoffzentrums (kurz: RWZ) zu finden (die Gesteinshüttenkunde verblieb im Peter-Tunner-Gebäude), im etwa 150 m Luftlinie zum RWZ situierten und auf die Forschungsbedürfnisse der Lehrstühle ausgerichteten Impulszentrum für Rohstoffe (kurz: IZ Rohstoffe) werden seit der Eröffnung im Mai 2011 schwerpunktmäßig forschungsrelevante Aktivitäten aller vier Lehrstühle betrieben. Der Rohstoffbereich hat diesen sich ankündigenden Prozess auch dazu genutzt, einige weitreichende Änderungen vorzunehmen. So wurden die drei Diplomstudien "Bergwesen“, "Gesteinshüttenwesen" und "Markscheidewesen" zu einem Bachelorstudium - welches zunächst "Natural Resources" hieß und dzt. mit dem Namen "Rohstoffingenieurwesen" angeboten wird - und zwei Masterstudien - "Rohstoffgewinnung und Tunnelbau" und "Rohstoffverarbeitung " - zusammengeführt.

Mit der Teilnahme der MUL am EIT RawMaterials, welches europaweit 125 Institutionen umfasst, hat der Rohstoffbereich der MUL zweifelsohne eine deutliche Aufwertung erfahren. Die MUL nimmt in diesem Forschungsnetzwerk eine äußerst aktive Rolle ein, was sich auch in der Zuerkennung eines RIC Leoben (Regional Innovation Center Leoben), zuständig für die Region Süd- und Südost-Europa, ableiten lässt. Gegenwärtig sind im RIC Leoben 14 Personen in Vollzeit angestellt, die dem Lehrstuhl für Bergbaukunde, Bergtechnik und Bergwirtschaft zugeordnet sind und sich im Rahmen des EIT RawMaterials schwerpunktmäßig mit den drei thematischen Säulen "Raw Materials Innovation", "Education“ und „ESEE (East and South East Europe) Regional Development" befassen.

Mit dem ZaB (Zentrum am Berg) steht dem Lehrstuhl für Subsurface Engineering eine Einrichtung für die Forschung und Lehre zur Verfügung, die sowohl national wie auch international einzigartig ist. Diese als eigenes Department eingerichtete Forschungseinrichtung steht unmittelbar vor der Fertigstellung und erfuhr durch die Einrichtung einer eigenen Professur für „Digitale Transformation im Tunnelbau" und zwei Habilitationsstellen auch personell eine wesentliche Aufwertung.

Auch in der Lehre haben die im Department vertretenen Lehrstühle vielfältige Aktivitäten entfaltet. Ausgehend vom siebensemestrigen Bachelorstudium „Rohstoffingenieurwesen“ (ein Semester verpflichtende Praxis) werden die beiden viersemestrigen Masterstudien "Rohstoffgewinnung und Tunnelbau“ und "Rohstoffverarbeitung" (ein Semester für die Masterarbeit) mit jeweils mehreren Schwerpunktfächern zur Spezialisierung angeboten. Darüber hinaus sind zwei internationale Masterstudien eingerichtet, der "International Master of Science in Advanced Mineral Resources Development" und der "International Master of Science in Building Materials and Ceramics". Zudem bietet das Department im Sinne des LifeLong-Learnings fünf Universitätslehrgänge "New Austrian Tunneling Method (NATM) - Master of Engineering“, "International Mining Engineer", "Rock Engineering for Deep Mines", "Rohstoffaufbereitung" und "Sprengtechnik" an, die gut angenommen werden. Schlussendlich sei vermerkt, dass die Lehrstühle auch in die Lehre für andere Studienrichtungen involviert sind, der Lehrstuhl für Aufbe- 
reitung und Veredlung beispielsweise in die Bachelor- und Masterstudien „Recyclingtechnik“.

\subsection{Der Lehrstuhl für Aufbereitung und Veredlung}

\subsubsection{Kurzer geschichtlicher Abriss}

Mit der Berufung von Prof. Bierbrauer, der seinen Dienst im Oktober 1930 (also ziemlich genau vor 90 Jahren) antrat, wurde die Lehrkanzel für Aufbereitung und Veredlung aus der Taufe gehoben. Bierbrauer verstand es, die traditionelle Eingrenzung der Aufbereitung zwischen dem Bergbau auf Erze und Kohlen einerseits und dem metallgewinnenden Hüttenwesen andererseits aufzubrechen und um das weite Feld der Steine und Erden einschließlich gewisser Teilbereiche der Weiterverarbeitung von mineralischen Rohstoffen zu ergänzen [1]. Eine ausführliche Würdigung der Persönlichkeit und des wissenschaftlichen Lebenswerkes von Prof. Bierbrauer ist in [2] zu finden.

Prof. Steiner trat seinen Dienst im Mai 1968 an. Konsequent setzte er den von Prof. Bierbrauer eingeschlagenen Weg einer apparativen und methodischen Abbildung der aufbereitungstechnischen Kleinversuchstechnik fort, indem er den Grundstock an Zerkleinerung-, Klassier- und Sortierapparaten durch Zukäufe, Zuwendungen und Eigenkonstruktionen nach umfangreichen Umbau- und Adaptierungsarbeiten im Rittinger-Gebäude komplettierte. Die Lehre war Steiner ein besonderes Anliegen und er nahm sich die dafür notwendige Zeit für eine praxisorientierte und zu vernetztem Denken anleitende Vermittlung des Lehrstoffes. In der Forschung erwarb er sich insbesondere in den Bereichen Flotationskinetik, Aufschlusskinetik und Zerkleinerungsforschung internationale Reputation. Anlässlich seines 75. Geburtstages haben der Lehrstuhl für Aufbereitung und Veredlung in Kooperation mit dem Fachausschuss für Aufbereitung im Bergmännischen Verband Österreichs die Festschrift "Aufbereitung in Österreich II" [3] herausgegeben, die das eindrucksvolle wissenschaftliche Vermächtnis von Prof. Steiner zusammenfasst. Eine ausführliche Würdigung der Persönlichkeit und des wissenschaftlichen Lebenswerkes von Prof. Steiner ist in [4] zu finden.
Seit September 2005 darf der Autor dieser Zeilen die Geschicke des Lehrstuhls für Aufbereitung und Veredlung leiten. In diesen Zeitraum seit 2005 fielen u. a.

- der Zusammenschluss rohstofforientierter Lehrstühle zu einem schlagkräftigen Department,

- die Planung, der Bau, die Besiedelung und die Inbetriebnahme eines modernsten Ansprüchen genügenden Aufbereitungstechnikums und Aufbereitungslabors im Impulszentrum für Rohstoffe sowie

- die Änderung der Studienarchitektur hin zu Bachelorund Masterstudien und damit die Chance zum Aufbau des Schwerpunktfaches Aufbereitung und Veredlung im Masterstudium Rohstoffverarbeitung wie auch

- der Aufbau des Universitätslehrganges Rohstoffaufbereitung.

\subsubsection{Die Lehre}

Status quo in der Lehre In Tab. 1 sind jene Lehrveranstaltungen (LV) ausgewiesen, die der Lehrstuhl für Aufbereitung und Veredlung für das Bachelorstudium "Rohstoffingenieurwesen“ (RIW) wie auch für die Bachelorstudien "Recyclingtechnik“ (RT), "Verfahrenstechnik des industriellen Umweltschutzes" (IU) und "Montanmaschinenbau“ (MB) sowie für die Masterstudien "Angewandte Geowissenschaften" (AG) und "Metallurgie" (MET) in ausschließlich deutscher Sprache anbietet.

Den überwiegenden Teil der Lehrveranstaltungen (LV) bietet der Lehrstuhl für Aufbereitung und Veredlung gemäß Tab. 2 für das Masterstudium „Rohstoffverarbeitung“ (RV) wie auch für die Masterstudien „Rohstoffgewinnung und Tunnelbau“ (RG\&T), „Recyclingtechnik“ (RT), „Verfahrenstechnik des industriellen Umweltschutzes" (IU), "Industrielle Energietechnik“ (IET), „Metallurgie“ (MET), „International Master of Science in Advanced Mineral Resources Development" (AMRD) und "International Master of Science in Building Materials and Ceramics" (BM\&C) in deutscher und englischer Sprache an.

Zudem hat der Lehrstuhl für Aufbereitung und Veredlung im Studienjahr 2013/14 einen Universitätslehrgang (ULG) Rohstoffaufbereitung ins Leben gerufen, der ein

\begin{tabular}{|c|c|c|c|c|c|}
\hline Lehrveranstaltungsbezeichnung & LV-Typ & SST & ECTS-Punkte & BSc-Studien & MSc-Studien \\
\hline Grundzüge der Aufbereitung & VO & 2,0 & 3,0 & RIW, RT & AG, MET \\
\hline Aufbereitungsverfahren und -anlagen & VO & 2,0 & 3,0 & RIW, RT & - \\
\hline Übungen zu Aufbereitungsverfahren und -anlagen & UE & 3,0 & 3,0 & RIW & - \\
\hline Probenahme und Vergleichmäßigung & IV & 3,0 & 4,0 & RT & $\mathrm{RV}, \mathrm{BM} \& \mathrm{C}$ \\
\hline Seminar Bachelorarbeit - Rohstoffingenieurwesen & SE & 6,0 & 7,5 & RIW & - \\
\hline Seminar Bachelorarbeit - Reyclingtechnik & SE & 6,0 & 7,5 & RT & - \\
\hline Einführung in das Rohstoffingenieurwesen & VO & 1,0 & 1,5 & RIW, MB & - \\
\hline Abfallaufbereitung & VO & 2,0 & 3,0 & RT, IU & - \\
\hline Summe & - & 25,0 & 32,5 & - & - \\
\hline
\end{tabular}




\begin{tabular}{|c|c|c|c|c|}
\hline Lehrveranstaltungsbezeichnung & LV-Typ & SST & ECTS-Punkte & MSc-Studien \\
\hline Theorie der Aufbereitungsprozesse & VO & 2,0 & 3,0 & $\mathrm{RV}, \mathrm{RT}, \mathrm{BM} \& \mathrm{C}$ \\
\hline Probenahme und Vergleichmäßigung & IV & 3,0 & 4,0 & $\mathrm{RV}, \mathrm{BM} \& \mathrm{C}$ \\
\hline $\begin{array}{l}\text { Planung, Instrumentierung und Verfahrenslenkung in Aufberei- } \\
\text { tungsanlagen }\end{array}$ & IV & 2,0 & 2,5 & $\mathrm{RV}$ \\
\hline Mineralogische Untersuchungen im Aufbereitungslabor & IV & 2,0 & 2,5 & RV \\
\hline Aufbereitung von Industriemineralen & VO & 2,0 & 3,0 & $\mathrm{RV}, \mathrm{BM} \& \mathrm{C}$ \\
\hline Übungen zu Aufbereitung von Industriemineralen & UE & 3,0 & 3,0 & $\mathrm{RV}, \mathrm{BM} \& \mathrm{C}$ \\
\hline Flotation & VO & 1,0 & 1,5 & RV \\
\hline Erzaufbereitung & VO & 1,0 & 1,5 & RV, MET \\
\hline Übungen zu Erzaufbereitung & UE & 1,0 & 1,0 & $\mathrm{RV}$ \\
\hline Aufbereitung von Salzmineralen & VO & 1,0 & 1,5 & RV \\
\hline Aufbereitung von Energierohstoffen I & VO & 1,5 & 2,0 & RV, IET \\
\hline Aufbereitung von Energierohstoffen II & VO & 1,5 & 2,0 & RV, IET, BM\&C \\
\hline Übungen zu Aufbereitung von Energierohstoffen & UE & 1,5 & 2,0 & RT \\
\hline Bodenaufbereitung & VO & 1,0 & 1,5 & $\mathrm{RV}, \mathrm{RT}$ \\
\hline Aufbereitung von Baurohstoffen & VO & 1,0 & 1,5 & RV \\
\hline $\begin{array}{l}\text { Aufbereitung industrieller Reststoffe - Schlacken, Schlämme, } \\
\text { Stäube }\end{array}$ & VO & 1,0 & 1,5 & $\begin{array}{l}\text { RV, RT, IU, MET, } \\
\text { BM\&C }\end{array}$ \\
\hline Messtechnik und Automation in der Aufbereitung & IV & 2,0 & 2,5 & RV \\
\hline $\begin{array}{l}\text { Computerunterstützte Anlagenplanung in der Mineralaufberei- } \\
\text { tung }\end{array}$ & VO & 2,0 & 3,0 & RV \\
\hline $\begin{array}{l}\text { Übungen zu Computerunterstützte Anlagenplanung in der Mine- } \\
\text { ralaufbereitung }\end{array}$ & UE & 2,0 & 2,0 & $\mathrm{RV}$ \\
\hline Aufbereitungstechnische Projektstudie & SE & 2,5 & 3,5 & $\mathrm{RV}, \mathrm{BM} \& \mathrm{C}$ \\
\hline Aufbereitungstechnische Projektstudie (Modul 1 für RT) & SE & 3,0 & 3,5 & RT \\
\hline Aufbereitungstechnisches Seminar & SE & 2,0 & 2,0 & $\mathrm{RV}, \mathrm{BM} \& \mathrm{C}$ \\
\hline Aufbereitungstechnische Exkursion & EX & 1,0 & 1,0 & $\mathrm{RV}$ \\
\hline Aufbereitungstechnischer Laborbetrieb & VO & 2,0 & 3,0 & $\mathrm{RV}, \mathrm{BM} \& \mathrm{C}$ \\
\hline $\begin{array}{l}\text { Rechtliche, sicherheitstechnische und umweltrelevante Aspekte in } \\
\text { der Aufbereitung }\end{array}$ & VO & 2,0 & 3,0 & $\mathrm{RV}, \mathrm{RG} \& \mathrm{~T}, \mathrm{RT}$ \\
\hline $\begin{array}{l}\text { Aufbereitung von Sekundärbrennstoffen - spezielle Abfallaufbe- } \\
\text { reitung für IU }\end{array}$ & VO & 2,0 & 3,0 & IU \\
\hline Seminar Masterarbeit - Rohstoffverarbeitung & SE & 3,0 & 3,0 & RV \\
\hline Seminar Masterarbeit - Recyclingtechnik & SE & 3,0 & 3,0 & RV \\
\hline Seminar Masterarbeit - International Master of Science in AMRD & SE & 3,0 & 3,0 & AMRD \\
\hline Seminar Masterarbeit - International Master of Science in BMC & SE & 3,0 & 3,0 & $\mathrm{BM} \& \mathrm{C}$ \\
\hline Summe & - & 58,0 & 72,5 & - \\
\hline
\end{tabular}

VO Vorlesung, UE Übung, IV Integrierte Veranstaltung, SE Seminar, EX Exkursion

Angebot zur berufsbegleitenden Höherqualifizierung für Personen, die in der Rohstoffindustrie und im aufbereitungstechnischen Maschinen- und Anlagenbau arbeiten, darstellt. Die Teilnehmer*innen werden in 10, jeweils auf drei Tage anberaumten Modulen theoretisch und praktisch im Fachgebiet „Aufbereitung und Veredlung" weitergebildet. Der Universitätslehrgang erstreckt sich über zwei Semester und umfasst einen Arbeitsaufwand von insgesamt 60 ECTS-Punkten. Davon entfallen auf Lehrveranstaltungen 45 ECTS-Punkte (siehe Tab. 3), auf die schriftliche Projektarbeit 12 ECTS-Punkte und auf die abschließende kommissionelle Prüfung 3 ECTS-Punkte. Den Absolvent*innen des ULGs wird von der Montanuniversität Leoben die Be- zeichnung "Akademische Aufbereitungstechnikerin“ bzw. „Akademischer Aufbereitungstechniker" verliehen.

Absolvent*innenentwicklung Um einen Eindruck darüber zu bekommen, in welchen Branchen die Graduierten arbeiten bzw. in welchen Ländern sie ihrer Arbeit überwiegend nachgehen, erfolgte eine Auswertung der Aufzeichnungen zur Absolvent*innenentwicklung des Lehrstuhls für einen Zeitraum von 15 Jahren. In diesem Zeitraum graduierten 60 Absolvent*innen, also im Schnitt 4 Absolvent*innen pro Jahr. Der Anteil weiblicher Absolventinnen, es sind 10 an der Zahl, liegt damit bei knapp 17\%. Mit einer einzigen Ausnahme haben alle in Österreich maturiert. Es wurden 


\begin{tabular}{|c|c|c|c|c|}
\hline Lehrveranstaltungsbezeichnung & LV-Typ & SST & ECTS-Punkte & Semester \\
\hline Grundlagen aus Physik, Chemie, Mineralogie und Geologie & VO & 3,0 & 4,5 & 1 \\
\hline $\begin{array}{l}\text { Grundlagen der Aufbereitungskunde I - Aufbereitungstechnische Grundpro- } \\
\text { zesse, Zerkleinern, Klassieren }\end{array}$ & VO & 3,0 & 4,5 & 1 \\
\hline $\begin{array}{l}\text { Grundlagen der Aufbereitungskunde II - Sortieren, Hilfsprozesse, Aufberei- } \\
\text { tungstechnische Schaltungen }\end{array}$ & VO & 3,0 & 4,5 & 1 \\
\hline $\begin{array}{l}\text { Aufbereitungstechnisches Rechnen - Bilanzierung, Teilungskurve, Beschrei- } \\
\text { bung von Verwachsungsverhältnissen }\end{array}$ & VO & 3,0 & 4,5 & 1 \\
\hline $\begin{array}{l}\text { Umweltschutz, Anlagen- und Prozesssicherheit - Anlagenüberwachung, } \\
\text { Anlagen- und Prozesssicherheit, Emissionen }\end{array}$ & VO & 3,0 & 4,5 & 1 \\
\hline $\begin{array}{l}\text { Aufbereitungstechnisches Laborpraktikum - Merkmalsklassenanalyse, auf- } \\
\text { bereitungstechnische Laboruntersuchungen }\end{array}$ & VO & 3,0 & 4,5 & 2 \\
\hline $\begin{array}{l}\text { Spezielle Aufbereitung primärer und sekundärer Rohstoffe I - Baurohstoffe, } \\
\text { Funktionsminerale, Instrumentierung und Verfahrenslenkung }\end{array}$ & VO & 3,0 & 4,5 & 2 \\
\hline $\begin{array}{l}\text { Spezielle Aufbereitung primärer und sekundärer Rohstoffe II - Abfallwirt- } \\
\text { schaft, Böden, Brennstoffe, Schlacken, Schlämme, Schrotte, Stäube }\end{array}$ & VO & 3,0 & 4,5 & 2 \\
\hline $\begin{array}{l}\text { Spezielle Aufbereitung primärer und sekundärer Rohstoffe III - Bindemittel, } \\
\text { Erze, Feuerfestrohstoffe, Rohstoff- und Beschaffungsmärkte }\end{array}$ & VO & 3,0 & 4,5 & 2 \\
\hline Exkursionen & EX & 3,0 & 4,5 & 2 \\
\hline Gesamtsumme & - & 30,0 & 45,0 & - \\
\hline
\end{tabular}

ausschließlich jene Absolvent*innen in die Betrachtung mit einbezogen, die das Schwerpunktfach "Aufbereitung und Veredlung“ im Masterstudium „Rohstoffverarbeitung“ absolviert haben.

Aus Abb. 1 lässt sich erkennen, dass in der Rohstoffindustrie $56 \%$ und im aufbereitungstechnischen Maschinenund Anlagenbau 20\% der Absolvent*innen ihren beruflichen Herausforderungen nachgehen und damit der überwiegende Anteil in den Kernbranchen der Aufbereitungstechnik tätig ist. Bezieht man die $7 \%$ der an der Montanuni-

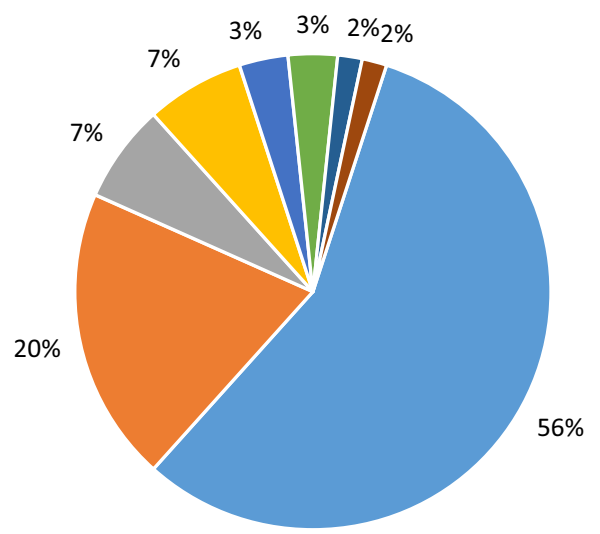
- Rohstoffunternehmen
- Maschinen- und Anlagenbau
- Montanuniversität Leoben
Fachfremd
- Dienstleistung
- Behörde
- HTL-Leoben
- Jobsuche

Abb. 1: Graduierte des Lehrstuhls, sortiert nach dem Merkmal „Branche“ in der sie arbeiten versität Leoben im Rahmen ihrer anwendungsorientierten Dissertationen wirkenden Graduierten in die Betrachtung ein, sind es deutlich mehr als $80 \%$.

Abb. 2 wiederum zeigt auf, dass mit $80 \%$ der größte Teil der Absolvent*innen in Österreich bzw. zumindest von Österreich aus seinen/ihren beruflichen Aktivitäten nachgeht und weitere $14 \%$ in der EU beschäftigt sind. Lediglich $6 \%$ sind in Übersee beschäftigt.

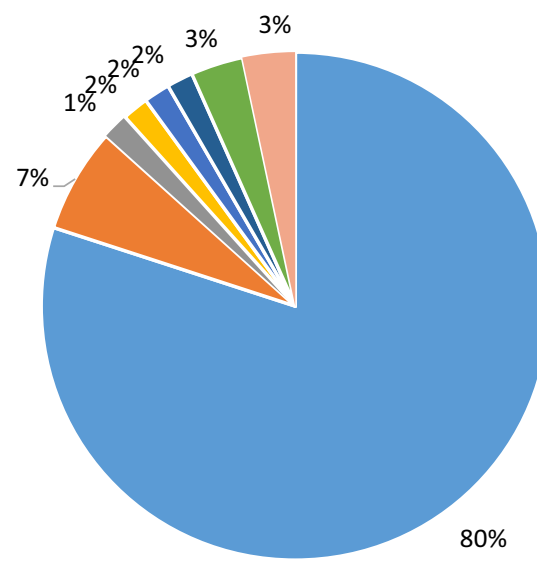

$$
\begin{array}{ll}
\square \text { Österreich } & \square \text { Deutschland } \\
\square \text { USA } & \square \text { Australien } \\
\square \text { Italien } & \square \text { Frankreich }
\end{array}
$$

Abb. 2: Graduierte des Lehrstuhls, sortiert nach dem Merkmal "Land“,in dem bzw. von dem aus sie ihrer Arbeit nachgehen 
Potenziale in der Lehre Die Absolvent*innen des Lehrstuhls werden von der Industrie gerne angestellt, die Rückmeldungen sind durchwegs positiv. Dies ist natürlich auch darin begründet, dass sich die in die Lehre des Lehrstuhls involvierten internen und externen Lehrbeauftragten der Lehre in Kleingruppen, also in einem sehr familiären Umfeld widmen und damit sehr individuell auf die Studierenden eingehen können. Zudem ist die überwiegende Anzahl der Studierenden des Masterstudiums in aufbereitungstechnische Labor- und Technikumsarbeit involviert.

In Zeiten sinkender Hörerzahlen wird es allerdings immer schwieriger, die mittlere Durchsatzrate von 4 Absolvent*innen an überwiegend inländischen Hörer*innen pro Jahr zu halten und daraus so etwas wie eine Existenzberechtigung abzuleiten. Der Lehrstuhl hat sich daher dazu entschlossen, an einer Antragstellung zur Einrichtung eines „Erasmus Mundus Joint Master Degree“-Studiums in "Sustainable Mineral and Metal Processing Engineering" (Kurzbezeichnung; PROMISE) gemeinsam mit den Universitäten Oulu (Finnland), Santa Maria (Chile) und Zagreb (Kroatien) mitzuwirken und sich dabei mit Partneruniversitäten und Forschungsinstitutionen schwerpunktmäßig in Europa und teilweise auch in Übersee besser zu vernetzen. Dieses englischsprachige Masterstudium für weltweit gefragte Aufbereiter*innen soll ganz bewusst ein ergänzendes Angebot zum etablierten Masterstudium "Rohstoffverarbeitung" darstellen, welches überwiegend Ingenieur*innen für die österreichische bzw. europäische Rohstoffindustrie und den österreichischen bzw. europäischen Maschinen- und Anlagenbau bereitstellt. In vier Durchgängen des zunächst auf sechs Jahre ausgelegten Masterstudiums könnten bis zu 88 internationale Studierende für ein bis zwei Semester (das 2. bzw. 4. Semester) nach Leoben gelockt werden.

Parallel dazu werden gegenwärtig Überlegungen angestellt, den im D-A-CH-Raum etablierten Universitätslehrgang „Rohstoffaufbereitung”, dessen 4. Jahrgang mit 17 Teilnehmer*innen kürzlich gestartet wurde, auch für ein internationales Publikum zu organisieren. Diesen Universitätslehrgang auf vier Semester auszubauen und mit einem „Master of Engineering in Mineral Processing“ abschließen zu lassen, ist eine weitere Überlegung.

\subsubsection{Die Forschung}

Status quo in der Forschung Die Forschungsaktivitäten der Leobener Aufbereiterinnen und Aufbereiter sind vielfältiger Natur und reichen gegenwärtig von Themen der klassischen Mineralaufbereitung und Ausdehnung der Technik auf die Reststoffaufbereitung, über Apparate- und Verfahrensentwicklungen bis hin zu speziellen Fragestellungen wie der Funktionalisierung von Rohstoffen oder der Aufbereitung kontaminierter Böden. Einen guten Überblick der Forschungsaktivitäten (und Entwicklungstendenzen) geben der Beitrag von Flachberger und Böhm [5] und die abermals in Kooperation mit dem Fachausschuss für Aufbereitung im Bergmännischen Verband Österreichs herausgegebene Festschrift "Aufbereitung in Österreich III" [6].
In der nachfolgenden Tab. 4 sind die Themenstellungen bereits abgeschlossener Dissertationen der vergangenen 15 Jahre übersichtlich zusammengestellt.

Bei genauerer Durchsicht wird deutlich, dass etwa die Hälfte der Themenstellungen der „klassischen Mineralaufbereitung", etwa ein Drittel der "Sekundärrohstoffaufbereitung" und der verbleibende Rest dem vergleichsweise jungen Forschungsschwerpunkt der „Funktionsmineralaufbereitung“ zuzuordnen sind. Die Themen leiten sich nicht zuletzt von den vielfältigen Herausforderungen der überwiegend europäischen Industriepartner ab.

Die klassische Mineralaufbereitung war und ist somit die tragende Säule der Forschungsaktivitäten und es soll dieser Forschungsschwerpunkt auch künftig in der ganzen ingenieurmäßigen Vielfalt abgedeckt und weiterentwickelt werden.

Die Bedeutung der Lehrstuhlaktivitäten rund um den Forschungsschwerpunkt Sekundärrohstoffaufbereitung nimmt stetig zu. Gegenwärtig wird am Lehrstuhl etwa die erneute Nutzung (Verwertung) von Reststoffströmen (oder relevanten Teilen davon) durch Rückführung in den Produktionsprozess oder für anderweitige Einsatzmöglichkeiten durch Anwendung moderner Aufbereitungsverfahren erforscht. Derartige Reststoffquellen, die unter Anwendung physikalischer Gesetzmäßigkeiten auf Aufbereitbarkeit geprüft bzw. darauf aufbauende Prozesse entwickelt werden, sind insbesondere Halden und Schlammteiche, Schlacken, Industriestäube, gebrauchte Feuerfestprodukte, Baurestmassen, schwer aufbereitbare Verbundwerkstoffe, Fraktionen der Elektronikschrottaufbereitung oder des Autorecyclings. Beispielhaft seien hier die Forschungsaktivitäten im Rahmen von K1Met und dem COMET-Projekt COMMBY genannt. Im Rahmen von K1Met werden neue Vorbereitungsverfahren entwickelt, um die Anwendung von klassischen Aufbereitungsprozessen auf feinst verwachsene Sekundärrohstoffe zu ermöglichen (z. B. die Konditionierung von $\mathrm{C}_{2} \mathrm{~S}$-haltigen Schlacken zur Eisenrückgewinnung durch umweltfreundliche, wirtschaftliche Laugungsverfahren und dazugehörige Prozessentwicklung). Das von drei Lehrstühlen der MUL getragene COMET-Projekt „COMMBY“ wurde ins Leben gerufen, um die Verwertung von metallurgischen Nebenprodukten zu forcieren. In Zusammenarbeit mit sieben Industriepartnern werden verschiedene Reststoffe beurteilt und entsprechende Prozesse entwickelt und optimiert. Ein weiteres wichtiges Ziel dieses Projekts ist es, ein Beurteilungsschema für sekundäre Rohstoffe zu entwickeln.

Unter Funktionsmineralaufbereitung wird das maßgeschneiderte Aufbereiten, Veredeln bzw. Konditionieren von Rohstoffen für die vielfältigen funktionellen Anwendungen verstanden, etwa den Einsatz von Füllstoffen in Kunststoffen zur Verbesserung der Compound-Eigenschaften. Diese Forschungsinitiative hat sich aufgrund der fächerübergreifenden Zusammenarbeit von Lehrstühlen der Montanuniversität Leoben-u. a. mit den Lehrstühlen des Departments für Kunststofftechnik - ergeben.

Promovent*innenentwicklung Für die Promovent*innen des Lehrstuhls erfolgte die gleiche Auswertung wie für die Graduierten, abermals sortiert nach den Merkmalen 


\begin{tabular}{|c|c|c|c|c|}
\hline $\begin{array}{l}\text { Lfd. Nr. } \\
\text { (seit } \\
\text { 1929) }\end{array}$ & Name & Titel der Dissertation & $\begin{array}{l}\text { Anstellungs- } \\
\text { verhältnis }\end{array}$ & $\begin{array}{l}\text { Ab- } \\
\text { schluss }\end{array}$ \\
\hline 28 & $\begin{array}{l}\text { GIGACHER, } \\
\text { Walter }\end{array}$ & $\begin{array}{l}\text { Elektrokinetische Messungen an Quarzkörnungen auf der Grundlage } \\
\text { von Strömungspotentialen }\end{array}$ & Intern & 2006 \\
\hline 29 & $\begin{array}{l}\text { ROTH, } \\
\text { Jürgen }\end{array}$ & $\begin{array}{l}\text { Submikronaufbereitung zur gezielten Beeinflussung von Rohstoffei- } \\
\text { genschaften }\end{array}$ & Extern & 2006 \\
\hline 30 & $\begin{array}{l}\text { MANGELBERGER, } \\
\text { Thomas }\end{array}$ & $\begin{array}{l}\text { Grenzen und Optimierungsmöglichkeiten der Aufbereitung von Cal- } \\
\text { ciumcarbonat für den Einsatz in der Füllstoffindustrie }\end{array}$ & Extern & 2007 \\
\hline 31 & $\begin{array}{l}\text { BERNHART, } \\
\text { Wolfram }\end{array}$ & $\begin{array}{l}\text { Die Aufbereitung von Wolframerzen unter besonderer Berücksichti- } \\
\text { gung der flotativen Anreicherung von Scheelit }\end{array}$ & Extern & 2008 \\
\hline 32 & $\begin{array}{l}\text { OHRDORF, } \\
\text { Karl-Heinz }\end{array}$ & Zur Frage der alkalischen Aktivierung von Bentoniten & Extern & 2010 \\
\hline 33 & $\begin{array}{l}\text { STADTSCHNITZER, } \\
\text { Alfred }\end{array}$ & $\begin{array}{l}\text { Optimierung des Schwerspat-/Flussspatausbringens in der Aufberei- } \\
\text { tungsanlage Wolfach der Sachtleben Bergbau GmbH }\end{array}$ & Intern & 2010 \\
\hline 34 & $\begin{array}{l}\text { BRUNNMAIR, } \\
\text { Erwin }\end{array}$ & $\begin{array}{l}\text { Entwicklung und Modellierung eines neuen Hochleistungszyklons } \\
\text { zur Trennung von Feststoff/Gas-Gemischen }\end{array}$ & Extern & 2010 \\
\hline 35 & $\begin{array}{l}\text { VAN OMMEN, } \\
\text { Roman }\end{array}$ & $\begin{array}{l}\text { Optimization of Classification Results by Controlling of Hydrocyclo- } \\
\text { ne Process Parameters }\end{array}$ & Extern & 2011 \\
\hline 36 & $\begin{array}{l}\text { RICHTER, } \\
\text { Rüdiger }\end{array}$ & $\begin{array}{l}\text { Processing of mercury-contaminated wastes, particularly from for- } \\
\text { mer chlorine-alkali electrolysis facilities and acetaldehyde sites in } \\
\text { soil washing plants }\end{array}$ & Extern & 2011 \\
\hline 37 & $\begin{array}{l}\text { OBERRAUNER, } \\
\text { Andreas }\end{array}$ & $\begin{array}{l}\text { Nutzung der Elektroscheidung zur trockenen Aufbereitung fein- und } \\
\text { feinstdisperser Körnerschwärme }\end{array}$ & Intern & 2012 \\
\hline 38 & $\begin{array}{l}\text { BAUER-VASKO, } \\
\text { Christine }\end{array}$ & $\begin{array}{l}\text { Development of an Evaluation Method for Processing Bauxite Ores } \\
\text { to be Applied in the Refractory Industry }\end{array}$ & Intern & 2012 \\
\hline 39 & $\begin{array}{l}\text { ÖFNER, } \\
\text { Wolfgang }\end{array}$ & $\begin{array}{l}\text { Einsatzmöglichkeiten und -grenzen der aufbereitungstechnischen } \\
\text { Merkmalsklassenanalyse zur Charkaterisierung feinkörniger Rest- } \\
\text { stoffe der Eisen- und Stahlproduktion als Grundlage eines verbes- } \\
\text { serten Wiedereinsatzes }\end{array}$ & Intern & 2013 \\
\hline 40 & $\begin{array}{l}\text { MEISSNER, } \\
\text { Paul }\end{array}$ & $\begin{array}{l}\text { Entwicklung einer Labor-Walzentellermühle und Erfassung von Zer- } \\
\text { kleinerungskenngrößen }\end{array}$ & Intern & 2013 \\
\hline 41 & $\begin{array}{l}\text { CIRAR, } \\
\text { Kristin }\end{array}$ & $\begin{array}{l}\text { Weiterentwicklung eines Verfahrens zur Herstellung geschlossen- } \\
\text { zellig expandierter Perlite zur Verbesserung der funktionellen Eigen- } \\
\text { schaften in neuartigen Produktanwendungen }\end{array}$ & Intern & 2013 \\
\hline 42 & $\begin{array}{l}\text { STREICHER, } \\
\text { Christian }\end{array}$ & Beitrag zur Optimierung von Querstrom-Drehkorbsichtern & Intern & 2013 \\
\hline 43 & $\begin{array}{l}\text { KÖNIGSHOFER, } \\
\text { Sandra }\end{array}$ & $\begin{array}{l}\text { Research on the processing of spent magnesia carbon refractory } \\
\text { bricks for developing innovative recycling approaches }\end{array}$ & Intern & 2015 \\
\hline 44 & $\begin{array}{l}\text { NIIRANEN, } \\
\text { Kari }\end{array}$ & $\begin{array}{l}\text { Characterization of the Kiirunavaara iron ore deposit for mineral } \\
\text { processing with a focus on the high silica ore type B2 }\end{array}$ & Extern & 2015 \\
\hline 45 & $\begin{array}{l}\text { KRISCHEY, } \\
\text { Elke }\end{array}$ & $\begin{array}{l}\text { Verfahren zur Herstellung von alkalisch und organophil aktiviertem } \\
\text { Kalzium-Montmorillonit für den Einsatz in Polymer-Compounds }\end{array}$ & Intern & 2015 \\
\hline 46 & $\begin{array}{l}\text { HUBER, } \\
\text { Reinhold }\end{array}$ & $\begin{array}{l}\text { Zu besonderen Fragestellungen der Einsatzmöglichkeiten und -gren- } \\
\text { zen der sensorgestützten Sortiertechnik bei der Aufbereitung pri- } \\
\text { märer und sekundärer Rohstoffe }\end{array}$ & Extern & 2016 \\
\hline 47 & $\begin{array}{l}\text { SCHMID, } \\
\text { Andreas }\end{array}$ & $\begin{array}{l}\text { Entwicklung einer Radialspalt-Rotorschere zur Zerkleinerung von } \\
\text { metallischen Spänen als wesentlicher Beitrag zur Aufbereitung } \\
\text { hochwertiger Briketts für den Einsatz in der metallurgischen Indus- } \\
\text { trie }\end{array}$ & Intern & 2016 \\
\hline 48 & $\begin{array}{l}\text { HERRERA, } \\
\text { Diego }\end{array}$ & $\begin{array}{l}\text { Mineral Processing Technologies on Biomass Preparation for Bypro- } \\
\text { duct Benefit in Metallurgy }\end{array}$ & Intern & 2016 \\
\hline 49 & $\begin{array}{l}\text { MIRKOWSKA, } \\
\text { Monika }\end{array}$ & $\begin{array}{l}\text { Triboelectric charge behavior of minerals at microscale for under- } \\
\text { standing the triboelectrostatic separation process }\end{array}$ & Intern & 2016 \\
\hline 50 & $\begin{array}{l}\text { TSCHUGG, } \\
\text { Julia }\end{array}$ & $\begin{array}{l}\text { Evaluation of the Application of High Pulse Power Fragmentation in } \\
\text { Processing Industrial Minerals }\end{array}$ & Intern & 2018 \\
\hline 51 & $\begin{array}{l}\text { LÄMMERER, } \\
\text { Wolfgang }\end{array}$ & $\begin{array}{l}\text { Zu Fragen der Anwendung neuer Methoden zur Charakterisierung } \\
\text { und Aufbereitung von Grafiten }\end{array}$ & Intern & 2018 \\
\hline 52 & $\begin{array}{l}\text { PATSCHEIDER, } \\
\text { Stefan }\end{array}$ & $\begin{array}{l}\text { Zu Fragen einer Verbesserung der Aufbereitungseffizienz bei der } \\
\text { Metallaufbereitung aus primären und sekundären Ressourcen }\end{array}$ & Intern & 2018 \\
\hline
\end{tabular}




\begin{tabular}{|c|c|c|c|c|}
\hline \multicolumn{5}{|c|}{$\begin{array}{l}\text { TABELLE } 4 \\
\text { (Fortsetzung) }\end{array}$} \\
\hline $\begin{array}{l}\text { Lfd. Nr. } \\
\text { (seit } \\
\text { 1929) }\end{array}$ & Name & Titel der Dissertation & $\begin{array}{l}\text { Anstellungs- } \\
\text { verhältnis }\end{array}$ & $\begin{array}{l}\text { Ab- } \\
\text { schluss }\end{array}$ \\
\hline 53 & $\begin{array}{l}\text { WEINGRILL, } \\
\text { Georg-Ulrich }\end{array}$ & $\begin{array}{l}\text { Entwicklung eines Verfahrens zur kontrollierten Expansion von fein- } \\
\text { dispersen Perlitrohsanden }\end{array}$ & Intern & 2019 \\
\hline 54 & $\begin{array}{l}\text { KAMALI-MOAVENI, } \\
\text { Ali }\end{array}$ & Physical-Chemical Processing of LD-Slags & Intern & 2019 \\
\hline 55 & $\begin{array}{l}\text { LARISSEGGER, } \\
\text { Sonja }\end{array}$ & $\begin{array}{l}\text { Aufbereitung von synthetischem Kauster für den Einsatz in der Pa- } \\
\text { pier- und Zellstoffindustrie }\end{array}$ & Extern & 2019 \\
\hline 56 & $\begin{array}{l}\text { SCHWABL, } \\
\text { Daniel }\end{array}$ & $\begin{array}{l}\text { Weiterentwicklung eines Verfahrens zur nass-mechanischen Aufbe- } \\
\text { reitung von polyolefinreichen Reststoffen zur Marktreife }\end{array}$ & Intern & 2020 \\
\hline 57 & $\begin{array}{l}\text { SALZER, } \\
\text { Florian }\end{array}$ & $\begin{array}{l}\text { Entwicklung einer Anlage zur Abscheidung von Quecksilber aus dem } \\
\text { Zementklinker-Herstellprozess }\end{array}$ & Extern & 2020 \\
\hline
\end{tabular}

„Branche“ und „Land“ wie auch für einen Zeitraum von 15 Jahren.

Der Abb. 3 ist zu entnehmen, dass zwar die Anteile der Promovierten, die im Dienstleistungssektor bzw. an Universitäten arbeiten, zunehmen, jedoch die Rohstoffunternehmen wie auch der aufbereitungstechnische Maschinen- und Anlagenbau mit in Summe $64 \%$ nach wie vor dominante Rollen einnehmen.

Abb. 4 wiederum zeigt auf, dass nach wie vor $67 \%$ der Promovierten in Österreich und weitere 18\% ihren beruflichen Lebensmittelpunkt in der EU haben. Der Anteil der außerhalb der EU Beschäftigten liegt bei $12 \%$.

Potenziale in der Forschung Wie der nachfolgenden Tab. 5 zu entnehmen ist, bleiben die Forschungsaktivitäten des Lehrstuhls auf die Technik des Zerkleinerns, Trennens nach physikalisch-chemischen Eigenschaften (fest/fest, fest/flüssig und fest/gasförmig) sowie des Agglomerierens fokussiert. Aufbereiter*innen sind und bleiben Trenntechniker*innen, ihre Passion liegt in der Verfügbarmachung

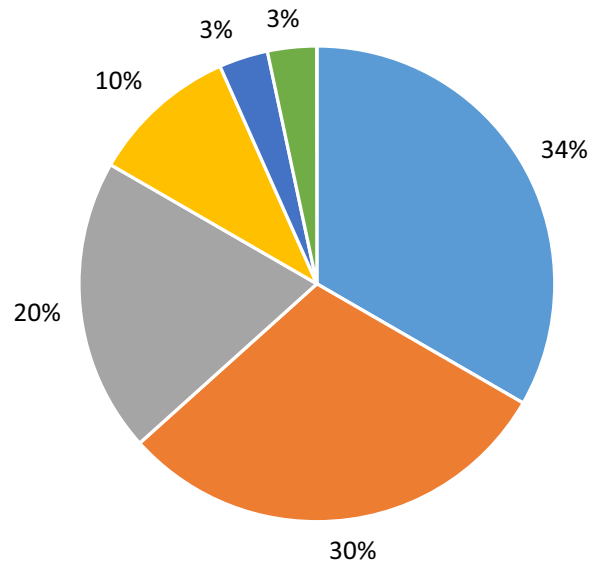

$$
\begin{aligned}
& \text { - Rohstoffunternehmen } \\
& \text { - Dienstleistung } \\
& \text { - Fachfremd }
\end{aligned}
$$$$
\text { - Maschinen- und Anlagenbau }
$$$$
\text { - Universität }
$$$$
\text { Jobsuche }
$$

Abb. 3: Promovierte des Lehrstuhls, sortiert nach dem Merkmal „Branche", in der sie arbeiten von Wertstoffen, sei es aus bergmännisch gewonnenen Rohgutarten oder aus diversen (industriellen) Reststoffen.

$\mathrm{Zu}$ der von Ass.-Prof. Dr. mont. Andreas Böhm geleiteten Arbeitsgruppe „Erz- und Schlackeaufbereitung“ und deren aktuellen wie auch künftigen Forschungsaktivitäten verweist der Verfasser auf die, diesem Artikel nachfolgende Veröffentlichung von Böhm et al. [7].

In der vom Verfasser geleiteten Arbeitsgruppe „Industriemineralaufbereitung und Rohstofffunktionalisierung" hat sich ein gewisser Schwerpunkt auf "Aufbereitungsprozesse in trockener Betriebsweise" herauskristallisiert, der auch in Zukunft einen großen Rahmen einnehmen wird. In den, diesem Artikel nachfolgenden Veröffentlichungen von Ohrdorf et al. [8], Gehringer et al. [9], Spieß et al. [10], Simon et al. [11] und Falkner et al. [12] werden einige aktuelle Forschungsthemen des Lehrstuhls in unterschiedlichen Reifestadien präsentiert.

Des Weiteren geht aus den in Anbahnung befindlichen Dissertationen deutlich hervor, dass ein großes Forschungspotential in der Digitalisierung aufbereitungstechnischer Prozesse und Verfahren liegt und dies in den kom-

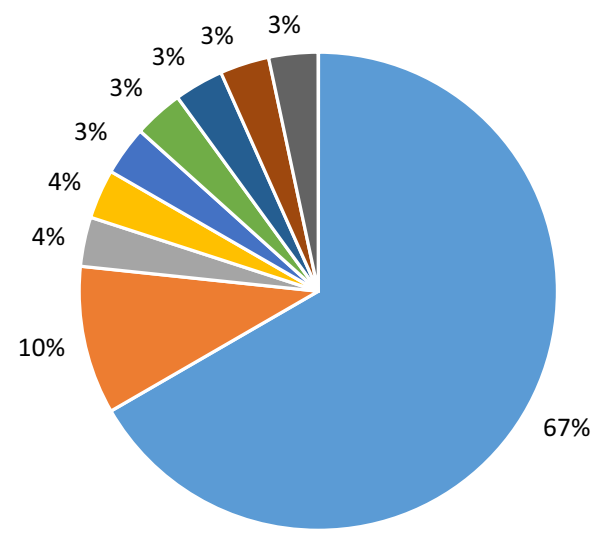
Österreich
- Deutschland
- Italien
- Schweden
Ecuador
Malaysien
- Jobsuche
- Vereinigte Arab. Emeriate
- Indien

Abb. 4: Promovierte des Lehrstuhls, sortiert nach dem Merkmal "Land" in dem bzw. von dem aus sie ihrer Arbeit nachgehen 


\begin{tabular}{|c|c|c|c|c|}
\hline $\begin{array}{l}\text { Lfd. Nr. } \\
\text { (seit 1929) }\end{array}$ & Name & Titel der Dissertation & $\begin{array}{l}\text { Anstellungs- } \\
\text { verhältnis }\end{array}$ & $\begin{array}{l}\text { Ab- } \\
\text { schluss }\end{array}$ \\
\hline 58 & $\begin{array}{l}\text { SCHWARZ, } \\
\text { Hubert Andreas }\end{array}$ & Innovative Aufbereitungsmethoden für Verbundstoffe & Intern & $\begin{array}{l}\text { In End- } \\
\text { phase }\end{array}$ \\
\hline 59 & $\begin{array}{l}\text { DONINGER, } \\
\text { Georg }\end{array}$ & $\begin{array}{l}\text { Untersuchungen zur Weiterentwicklung der Wirbelstrom-Sortier- } \\
\text { technik }\end{array}$ & Intern & $\begin{array}{l}\text { In End- } \\
\text { phase }\end{array}$ \\
\hline 60 & $\begin{array}{l}\text { GEHRINGER, } \\
\text { Sabrina }\end{array}$ & $\begin{array}{l}\text { Zu Fragen einer kontrollierten Aufladung von Mineraloberflä- } \\
\text { chen für eine erfolgreiche Trennung im elektrostatischen Feld }\end{array}$ & Intern & $\begin{array}{l}\text { In End- } \\
\text { phase }\end{array}$ \\
\hline 61 & $\begin{array}{l}\text { PLOCHBERGER, } \\
\text { Thomas }\end{array}$ & $\begin{array}{l}\text { Advancing fine grinding technologies for the mineral processing } \\
\text { industry }\end{array}$ & Extern & Laufend \\
\hline 62 & $\begin{array}{l}\text { LUCKENEDER, } \\
\text { Christoph }\end{array}$ & $\begin{array}{l}\text { Zu Fragen der Aufbereitung qualitativ hochwertiger, funktio- } \\
\text { neller Talkkonzentrate unter besonderer Berücksichtigung einer } \\
\text { trockenen Betriebsweise }\end{array}$ & Intern & Laufend \\
\hline 63 & $\begin{array}{l}\text { FALKNER, } \\
\text { Christoph }\end{array}$ & $\begin{array}{l}\text { Möglichkeiten und Grenzen der Simulation in der Brech- und } \\
\text { Siebtechnologie }\end{array}$ & Extern & Laufend \\
\hline 64 & $\begin{array}{l}\text { MAROUSEK, } \\
\text { Lukas }\end{array}$ & $\begin{array}{l}\text { Zu Fragen der Charakterisierung und Aufarbeitung metallhalti- } \\
\text { ger Rest- und Kreislaufstoffe aus aufbereitungstechnischer Sicht }\end{array}$ & Intern & Laufend \\
\hline 65 & $\begin{array}{l}\text { SPIEß, } \\
\text { Valentin }\end{array}$ & $\begin{array}{l}\text { Erarbeitung eines neuen Verfahrenskonzeptes zur Erzeugung } \\
\text { innovativer Mineralprodukte aus sekundären Rohstoffquellen }\end{array}$ & Intern & Laufend \\
\hline 66 & $\begin{array}{l}\text { SIMON, } \\
\text { Oliver }\end{array}$ & $\begin{array}{l}\text { Aufbereitung der bei der Kabelschrott-Verarbeitung anfallenden } \\
\text { Mischkunststofffraktion zu marktfähigen Produkten }\end{array}$ & Intern & Laufend \\
\hline 67 & $\begin{array}{l}\text { PREM, } \\
\text { Dominic }\end{array}$ & $\begin{array}{l}\text { Zu Fragen der Sulfidflotation in der Industriemineralaufberei- } \\
\text { tung }\end{array}$ & Intern & $\begin{array}{l}\text { In Anbah- } \\
\text { nung }\end{array}$ \\
\hline 68 & $\begin{array}{l}\text { KEMPER, } \\
\text { Dietmar }\end{array}$ & $\begin{array}{l}\text { Autonomes Brechen bei mobilen Zerkleinerungsaggregaten mit } \\
\text { Fokus auf eine energie- und qualitäts-optimierte Betriebsweise }\end{array}$ & Intern & $\begin{array}{l}\text { In Anbah- } \\
\text { nung }\end{array}$ \\
\hline 69 & $\begin{array}{l}\text { KIRCHNER, } \\
\text { Rupert }\end{array}$ & $\begin{array}{l}\text { Datenbasierte Modellierung und Regelung trockener Mahl-/ } \\
\text { Sicht-Kreisläufe }\end{array}$ & Extern & $\begin{array}{l}\text { In Anbah- } \\
\text { nung }\end{array}$ \\
\hline 70 & $\begin{array}{l}\text { REISINGER, } \\
\text { Florian }\end{array}$ & $\begin{array}{l}\text { "Smart Granulation" - Sensorunterstützte Regelungskonzepte } \\
\text { für Granulierprozesse mittels Pelletierteller }\end{array}$ & Extern & $\begin{array}{l}\text { In Anbah- } \\
\text { nung }\end{array}$ \\
\hline
\end{tabular}

menden Jahren in vielfältigen (auch fachübergreifenden) Forschungsaktivitäten seinen Niederschlag finden wird.

Die Forschungsaktivitäten, denen sich der Lehrstuhl auch in Zukunft verpflichtet fühlt, können somit wie folgt zusammengefasst werden:

- Weiterentwicklung der aufbereitungsrelevanten (Mineral-)Phasenanalytik, z.B. neue Schnellanalyseverfahren für große Mengen

- Verbesserung der Energieeffizienz in der Zerkleinerung und Weiterentwicklung von Zerkleinerungsmaschinen und -prozessen

- Weiterentwicklung der Aufbereitungstechnik in trockener Betriebsweise

- Rohstofffunktionalisierung

- Übertragung des systematischen aufbereitungstechnischen Methodenmixes zur Rohgutanalytik und etablierter aufbereitungstechnischer Prozesse auf das Gebiet der Sekundärrohstoff-Aufbereitung (z. B. Flotation von Sekundärrohstoffen)

- Digitalisierung von Aufbereitungsprozessen

- Mathematische Erfassung von Trenn- und Zerkleinerungsprozessen

Funding. Open access funding provided by Montanuniversität Leoben.
Open Access Dieser Artikel wird unter der Creative Commons Namensnennung 4.0 International Lizenz veröffentlicht, welche die Nutzung, Vervielfältigung, Bearbeitung, Verbreitung und Wiedergabe in jeglichem Medium und Format erlaubt, sofern Sie den/die ursprünglichen Autor(en) und die Quelle ordnungsgemäß nennen, einen Link zur Creative Commons Lizenz beifügen und angeben, ob Änderungen vorgenommen wurden.

Die in diesem Artikel enthaltenen Bilder und sonstiges Drittmaterial unterliegen ebenfalls der genannten Creative Commons Lizenz, sofern sich aus der Abbildungslegende nichts anderes ergibt. Sofern das betreffende Material nicht unter der genannten Creative Commons Lizenz steht und die betreffende Handlung nicht nach gesetzlichen Vorschriften erlaubt ist, ist für die oben aufgeführten Weiterverwendungen des Materials die Einwilligung des jeweiligen Rechteinhabers einzuholen.

Weitere Details zur Lizenz entnehmen Sie bitte der Lizenzinformation auf http://creativecommons.org/licenses/by/4.0/deed.de.

\section{Literatur}

1. Steiner, H.J.: Gründung und Entwicklung des Institutes für Aufbereitung und Veredlung bis zum Jahre 2005. In: Aufbereitung in Österreich, Festschrift anlässlich des 80-jährigen Bestehens des Lehrstuhls für Aufbereitung und Veredlung der Montanuniversität Leoben, ISBN978-3-200-02207-2, Leoben: Eigenverlag, 2010, pp 203-209

2. Steiner, H.J.: Zur Erinnerung an o. Prof. Dr.-Ing. Ernst Bierbrauer (1896-1973), BHM Berg- und Hüttenmännische Monatshefte 126 (1981), Heft 8, pp 315-320

3. Flachberger, H. et al. (Hrsg.): Aufbereitung in Österreich II - Festschrift anlässlich des 75. Geburtstages von Em.O.Univ.-Prof. Dipl.- 
Ing. Dr. mont. Hans Jörg Steiner, Ordinarius des Institutes für Aufbereitung und Veredlung der Montanuniversität Leoben in den Jahren 1968 bis 2005, ISBN 978-3-200-02738-1, Leoben: Eigenverlag, 2012

4. Flachberger, H.: Das Wirken von Em.O.Univ.-Prof. Dipl.-Ing. Dr. mont. Hans Jörg Steiner aus Sicht eines seiner Schüler und des Nachfolgers, BHM Berg- und Hüttenmännische Monatshefte 157 (2012), Heft 6/7, pp 225-229

5. Flachberger, H.; Böhm, A.: Entwicklungstendenzen im Fachgebiet Aufbereitung und Veredlung, BHM Berg- und Hüttenmännische Monatshefte 2015, Heft 10-11, pp 465-474

6. Flachberger, H. et. al. (Hrsg.): Aufbereitung in Österreich III - Dekadenbericht 2005 bis 2015 des Lehrstuhls für Aufbereitung und Veredlung der Montanuniversität Leoben, ISBN 978-3-200-04306-0, Leoben: Eigenverlag, 2015

7. Böhm A.; Mayer M.; Ressl G.: The 3rd Level - Entwicklung der Feinstzerkleinerung und Ausbau der Flotation am Lehrstuhl für Aufbereitung und Veredlung, BHM Berg- und Hüttenmännische Monatshefte 165 (2020), Heft 10, pp ...

8. Ohrdorf, K.H.; Birr, T.; Flachberger, H.: Einarbeitung von Nanoclay in Polyolefine mittels Planetwalzenextruder, BHM Berg- und Hüttenmännische Monatshefte 165 (2020), Heft 10, pp ...
9. Gehringer, S.; Luckeneder, C.; Flachberger, H.: Investigations on the triboelectrostatic charging behaviour and the triboelectrostatic sortability of different oxides, BHM Berg- und Hüttenmännische Monatshefte 165 (2020), Heft 10, pp...

10. Spieß, V.; Theiss, J.; Flachberger, H.: Erarbeitung eines neuen Verfahrenskonzeptes zur Erzeugung innovativer Mineralprodukte aus sekundären Rohstoffquellen, BHM Berg- und Hüttenmännische Monatshefte 165 (2020), Heft 10, pp ...

11. Simon, O.; Schwarz, H.A.; Flachberger, H.: Aufbereitung der bei der Kabelschrott-Verarbeitung anfallenden Mischkunststofffraktion zu marktfähigen Produkten, BHM Berg- und Hüttenmännische Monatshefte 165 (2020), Heft 10, pp ...

12. Falkner, P.; Niederhageböck, B.; Ton, J.; Gaggl, M.; Flachberger, H.: Optimierung eines Vorsiebes am Beispiel Active Grid ${ }^{\circledR}$ von RUBBLE MASTER mittels Simulation, BHM Berg- und Hüttenmännische Monatshefte 165 (2020), Heft 10, pp ...

Hinweis des Verlags. Der Verlag bleibt in Hinblick auf geografische Zuordnungen und Gebietsbezeichnungen in veröffentlichten Karten und Institutsadressen neutral. 